\title{
Laminar flame speed and shock-tube multi-species laser absorption measurements of Dimethyl Carbonate oxidation and pyrolysis near $1 \mathrm{~atm}$
}

\author{
T. Atherley ${ }^{1 *}$, S. de Persis ${ }^{2,3}$, N. Chaumeix ${ }^{2}$, Y. Fernandes ${ }^{2,3,4}$, A. Bry ${ }^{4}$, A. Comandini ${ }^{2}$, O. \\ Mathieu $^{1}$, S. Alturaifi ${ }^{1}$, C. R. Mulvihill ${ }^{1}$, E. L. Petersen ${ }^{1}$ \\ ${ }^{1}$ J. Mike Walker '66 Department of Mechanical Engineering, Texas A\&M University, College \\ Station, TX, USA \\ ${ }^{2}$ Institut de Combustion, Aérothermique, Réactivité et Environnement, CNRS Orléans, Orléans, \\ France \\ ${ }^{3}$ Université d'Orléans, Orléans, France \\ ${ }^{4}$ CEA-DAM Le Ripault, F-37260 Monts, France
}

\begin{abstract}
:
Dimethyl Carbonate (DMC) is a carbonate ester that can be produced in an environmentfriendly way from methanol and $\mathrm{CO}_{2}$. DMC is one of the main components of the flammable electrolyte used in Li-ion batteries, and it can also be used as a diesel fuel additive. Studying the combustion chemistry of DMC can therefore improve the use of biofuels and help developing safer Li-ion batteries. The combustion chemistry of DMC has been investigated in a limited number of studies. The aim of this study was to complement the scarce data available for DMC combustion in the literature. Laminar flame speeds at $318 \mathrm{~K}, 363 \mathrm{~K}$, and $463 \mathrm{~K}$ were measured for various equivalence ratios (ranging from 0.7 to 1.5 ) in a spherical vessel, greatly extending the range of conditions investigated. Shock tubes were used to measure time histories of $\mathrm{CO}$ and $\mathrm{H}_{2} \mathrm{O}$ using tunable laser absorption for the first time for DMC. Characteristic reaction times were also measured through $\mathrm{OH}^{*}$ emission. Shock-tube spectroscopic measurements were performed under dilute conditions, at three equivalence ratios (fuel-lean, stoichiometric, and fuel-rich) between 1260 and $1660 \mathrm{~K}$ near $1.3 \pm 0.2 \mathrm{~atm}$, and under pyrolysis conditions $(98 \%+)$ ranging from 1230 to $2500 \mathrm{~K}$ near $1.3 \pm 0.2 \mathrm{~atm}$. Laminar flame speed experiments were performed around atmospheric pressure. Detailed kinetics models from the literature were compared to the data, and it was found that none are capable of predicting the data over the entire range of conditions investigated. A numerical analysis was performed with the most accurate model, underlining the need to revisit at least 3 key reactions involving DMC.
\end{abstract}

Keywords: Dimethyl Carbonate, laminar flame speed, oxidation, pyrolysis, laser absorption, shock tubes

Corresponding author:

Tatyana Atherley

Email: tatyana.atherley@tamu.edu

Preprint submitted to Proceedings of the Combustion Institute 


\section{Introduction}

Dimethyl Carbonate $\left(\mathrm{CH}_{3} \mathrm{OCOOCH}_{3}, \mathrm{DMC}\right)$ is a promising biodiesel which can be directly synthesized from $\mathrm{CO}_{2}$ and methanol [1]. When used as an additive, results show a reduction in soot emissions without penalty on the engine's thermal efficiency or $\mathrm{NO}_{\mathrm{x}}$ emissions $[2,3]$. DMC is also a major component of Li-ion battery $(\mathrm{LiB})$ electrolytes (a flammable mixture of linear and cyclic carbonates [4]), allowing Lithium salt ions to flow between the cathode and the anode. The flammability of the electrolyte has led to many fire incidents, ranging from cell phones to airplanes, due to flaws in the design or fabrication processes or physical damages of the LiB [4]. With the rapidly growing number of electric vehicles on the roads, exposing large LiB to mechanical damage, and the rising use of mobile/portable devices, it is important to understand the combustion chemistry of LiB electrolyte components, to work towards reducing their flammability. To the best of the authors' knowledge, there are only few articles investigating the fundamental combustion chemistry of the carbonate solvents used in Li-ion batteries [5, 6].

The present study aims to do so by experimentally investigating DMC combustion chemistry, with an assortment of important combustion property measurements, such as ignition delay time and laminar flame speed, and more fundamental experiments such as laser-absorption measurements of $\mathrm{CO}$ and $\mathrm{H}_{2} \mathrm{O}$. Detailed kinetics mechanisms from Glaude et al. [7], Hu et al. [8], Sun et al. [9], and Alexandrino et al. [10] were compared to the data to assess their accuracy. Provided in this paper is a brief description of the experimental facilities, followed by the presentation of the experimental results and the comparison with the kinetics models. Finally, a numerical analysis was performed, leading to recommendations for model improvements. 


\section{Experimental Facilities}

\subsection{Shock Tubes}

Two shock tubes were used in this study, further referred to as the $\mathrm{CO} / \mathrm{OH}^{*}$ and $\mathrm{H}_{2} \mathrm{O}$ tubes. The $\mathrm{CO} / \mathrm{OH}^{*}$ tube is $6.1-\mathrm{m}$ long with a $4-\mathrm{m}, 10.8-\mathrm{cm}$ square driven section. The $\mathrm{H}_{2} \mathrm{O}$ tube has a 6.78-m-long driven section (16.2-cm ID) and a 3-m-long driver section. Both tubes are made of stainless-steel and have a single-diaphragm arrangement. Several piezoelectric pressure transducers (PCB P113A) are located along the driven sections to determine the incident shock wave velocity. A linear fit was used to extrapolate this velocity to the endwall location, which coupled with the one-dimensional shock relations and initial conditions, was used to determine the reflected-shock temperature $\left(\mathrm{T}_{5}\right)$ and pressure $\left(\mathrm{P}_{5}\right)$. These reflected-shock conditions have an estimated uncertainty of $\sim 1.0 \%[11]$.

Before each experiment, the driven section of the tubes were vacuumed to $\sim 10^{-5}$ Torr using a combination of roughing and turbomolecular pumps. All test mixtures were prepared manometrically in stainless-steel tanks using 0-10 Torr, 0-1000 Torr, and 0-13000 Torr pressure gauges. The DMC was provided by Sigma-Aldrich ${ }^{\circledR}(\geq 99 \%)$, while $\mathrm{O}_{2}$, Ar, and He were supplied by Praxair ${ }^{\circledR}, 99.999 \%$ purity. DMC was introduced in the mixing tank via a vial, after being degassed at least three times. Three equivalence ratios $(\phi), 0.5,1.0$, and 2.0, were studied between 1260 and $1660 \mathrm{~K}$ at $1.3 \pm 0.1 \mathrm{~atm}$, for mixtures diluted at $99.25 \%$. Pyrolysis of DMC was investigated as well, ranging from 1230 to $1560 \mathrm{~K}$ near $1.6 \pm 0.06$ atm with a dilution set to $99.75 \%$ for the $\mathrm{CO}$ measurement and 1980 to $2500 \mathrm{~K}$ near $1.1 \pm 0.09$ atm with $98 \%$ dilution for $\mathrm{H}_{2} \mathrm{O}$. The dilution levels used for this study were determined based on the allowable detection levels for proper and accurate laser absorption measurements. A high dilution level is also desirable as it mitigates the temperature changes induced by a mixture's reactivity (more on this below). Note 
that $20 \% \mathrm{He}$ was added (balance Ar) in the mixtures for $\mathrm{CO}$ measurements, due to possible vibrational relaxation effects [12]. A summary of all the experimental conditions investigated, along with the exact composition of the mixtures, can be seen in Table 1.

Table 1. Shock-tube conditions investigated during this study. Note that $\mathrm{OH}^{*}$ measurements were not taken for the pyrolysis case.

\begin{tabular}{|c|c|c|c|c|}
\hline Diagnostic & Mixture composition & Equiv. Ratio $(\phi)$ & $\mathrm{P}_{5}(\mathrm{~atm})$ & $\mathrm{T}_{5}(\mathrm{~K})$ \\
\hline \multirow{4}{*}{$\mathrm{CO} / \mathrm{OH}^{*}$} & $0.001071 \mathrm{DMC} / 0.006429 \mathrm{O}_{2} / 0.2 \mathrm{He} / 0.7925 \mathrm{Ar}$ & 0.5 & $1.28 \pm 0.05$ & $1316-1474$ \\
\cline { 2 - 5 } & $0.001875 \mathrm{DMC} / 0.005625 \mathrm{O}_{2} / 0.2 \mathrm{He} / 0.7925 \mathrm{Ar}$ & 1.0 & $1.33 \pm 0.08$ & $1323-1573$ \\
\cline { 2 - 5 } & $0.003 \mathrm{DMC} / 0.0045 \mathrm{O}_{2} / 0.2 \mathrm{He} / 0.7925 \mathrm{Ar}$ & 2.0 & $1.25 \pm 0.10$ & $1307-1642$ \\
\cline { 2 - 5 } & $0.0025 \mathrm{DMC} / 0.2 \mathrm{He} / 0.7975 \mathrm{Ar}$ & Pyrolysis & $1.57 \pm 0.06$ & $1232-1556$ \\
\hline \multirow{4}{*}{$\mathrm{H}_{2} \mathrm{O}$} & $0.001071 \mathrm{DMC} / 0.006429 \mathrm{O}_{2} / 0.9925 \mathrm{Ar}$ & 0.5 & $1.41 \pm 0.06$ & $1304-1546$ \\
\cline { 2 - 5 } & $0.001875 \mathrm{DMC} / 0.005625 \mathrm{O}_{2} / 0.9925 \mathrm{Ar}$ & 1.0 & $1.32 \pm 0.09$ & $1260-1573$ \\
\cline { 2 - 5 } & $0.003 \mathrm{DMC} / 0.0045 \mathrm{O}_{2} / 0.9925 \mathrm{Ar}$ & 2.0 & $1.37 \pm 0.07$ & $1354-1658$ \\
\cline { 2 - 5 } & $0.0201 \mathrm{DMC} / 0.9799 \mathrm{Ar}$ & Pyrolysis & $1.09 \pm 0.09$ & $1980-2499$ \\
\hline
\end{tabular}

\subsection{Laser Diagnostics}

The fundamental relation of the spectroscopic diagnostics used herein is the Beer-Lambert relation, $I / I_{0}=\exp \left(-k_{v} P X_{a b s} L\right)$, where $I$ and $I_{0}$ are the transmitted and incident laser intensities, respectively; $k_{v}$ the absorption coefficient $\left(\mathrm{cm}^{-1} \mathrm{~atm}^{-1}\right), P$ the pressure (atm), $X_{a b s}$ the species mole fraction, and $L$ the path length $(\mathrm{cm})$. The absorption coefficient, $k_{v}$ is the product of the linestrength (obtained from HITRAN 2004 [13]) and the lineshape (calculated using the Ar-broadening parameters from Ren et al. [14] for $\mathrm{CO}$ and Nagali et al. [15] for $\mathrm{H}_{2} \mathrm{O}$ ). The $\mathrm{CO}$ diagnostic was assembled using a quantum cascade laser with a $1.5-\mathrm{MHz}$ linewidth permitting access to the $\mathrm{R}(12)$ transition in the $1 \leftarrow 0$ band of $\mathrm{CO}$ at $4566.17 \mathrm{~nm}$. Throughout the experiments, the laser was centered at this $\mathrm{CO}$ transition line using a separate $\mathrm{CO} / \mathrm{Ar}$ absorption cell. Laser intensities were recorded using InSb detectors equipped with bandpass filters (centered at $4500 \mathrm{~nm}$, full width of $500 \mathrm{~nm}$ ) allowing a decrease in the broadband emission levels entering the detectors to $<0.3 \%$ of 
the absorbed signal. Minor $\mathrm{CO}_{2}$ absorption occurs at this wavelength, and the procedure described by Mulvihill et al. [16] was utilized to correct the $\mathrm{CO}$ time-histories. For further details on the $\mathrm{CO}$ diagnostics, please see [16] and references therein.

The $\mathrm{H}_{2} \mathrm{O}$ diagnostic uses a Toptica Photonics DL100L tunable diode laser set to 1388.140 nm, monitored using a Burleigh WA-1000 wavemeter. The incident and transmitted laser intensities were measured using two Newport 2317NF InGaAs detectors, fitted with a bandpass filter centered at $1388 \mathrm{~nm}$ with a full width, half maximum of $10 \mathrm{~nm}$. The laser beam path was purged with $\mathrm{N} 2$ to reduce the beam attenuation due to the air humidity. More details on the $\mathrm{H}_{2} \mathrm{O}$ diagnostic can be found in Mathieu et al. [17]. Note that an experiment with the laser turned off was performed, and no broadband emission was captured by the detector, even for the very high temperatures investigated for the pyrolysis case.

The change in $k_{v}$ with temperature was considered for each experiment, using the temperature change predicted by the Alexandrino et al. mechanism [10]. Further details on this correction method are described in recent work by the authors [16]. Estimated concentration level uncertainties are $\pm 5.6 \%$ for $\mathrm{H}_{2} \mathrm{O}$ and $\pm 3.8 \%$ for $\mathrm{CO}$.

\section{3. $\mathrm{OH}^{*}$ diagnostic}

A Hamamatsu 1P21 photomultiplier tube with a $307 \pm 10 \mathrm{~nm}$ bandpass filter was used to capture the emission from the $A^{2} \Sigma^{+} \rightarrow X^{2} \Pi$ transition of $\mathrm{OH}$. The characteristic reaction time used herein is the time to peak $\mathrm{OH}^{*}\left(\tau_{\text {peak }}\right)$ and is defined as the time where the maximum level of the $\mathrm{OH}^{*}$ signal was observed. Note that the test time is not long enough for the low-temperature cases to observe this peak and taking the time at the peak allows for characterization at higher temperatures, 
hence allowing a wider range of conditions. A typical $\tau_{\text {peak }}$ measurement is visible in Fig. 1 . The estimated $15 \%$ uncertainties for $\tau_{\text {peak }}$ comes mainly from the uncertainties in $\mathrm{T}_{5}$.

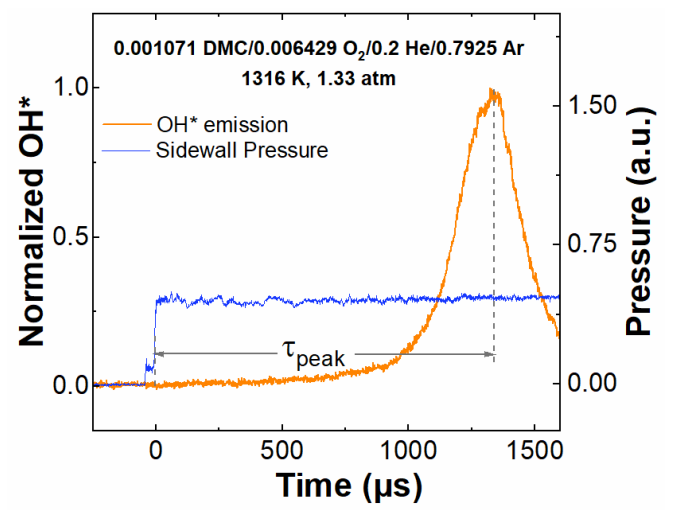

Figure 1. Typical sidewall pressure and $\mathrm{OH}^{*}$ emission signals recorded in this study with $\tau_{\text {peak }}$ definition.

\subsection{Laminar Flame Speed Vessel}

Laminar flame speeds $\left(S_{L, u}^{0}\right)$ were measured in a double-walled spherical vessel, with an internal diameter of $476 \mathrm{~mm}$. Between the two spheres, a heat transfer fluid heats the apparatus to the desired temperature $(318,363$, and $423 \mathrm{~K}$, with a $\pm 1 \mathrm{~K}$ homogeneity). The pressure during combustion was monitored using a piezo-electric transducer (Kistler 601A). Mixtures were sparkignited with two tungsten electrodes and a high-voltage generator, with an average energy delivery of $1.82 \mathrm{~mJ}$ with a standard deviation of $0.48 \mathrm{~mJ}$. Mixtures (initial pressure of $1.013 \mathrm{bar}$ ) were prepared inside the vessel using the partial pressure method with anhydrous DMC (Sigma Aldrich ${ }^{\circledR}$ $\geq 99 \%$ ) and dry air (Air Liquide, alphagaz $1^{\circledR}, 21 \% \mathrm{O}_{2} / 79 \% \mathrm{~N}_{2}, 99.999 \%$ purity). Pressures were measured using capacitance manometers (MKS, 0-100 and 0-1000 Torr). The spherical bomb is equipped with two opposing quartz windows (100-mm diameter, 50-mm thick), allowing for flame visualization using a Z-type schlieren apparatus. A white continuous lamp was used to illuminate 
the flame via two lenses and concave spherical mirrors. The schlieren images of the growing flame were recorded using a high-speed camera (PHANTOM V1610, $25000 \mathrm{fps}, 768 \times 768$ pixels ${ }^{2}$, fixed frame size).

Images were processed using a home-made Matlab code to obtain the flame radius $\left(\mathrm{R}_{\mathrm{f}}\right)$ as a function of time, from which the spatial (burned gas) flame speed $V_{S}=d_{R} / d t$ could be determined. A stretch correction to the velocity either using a non-linear or a linear extrapolation was used. For this study, all the unstretched spatial flame speeds between $10 \mathrm{~mm}$ and about $42 \mathrm{~mm}$ were extrapolated using the non-linear extrapolation from Ronney and Sivashinsky [18]. Since the spherical bomb allows the recording of large flames without any effect of the vessel, the laminar flame speed at zero-stretch is not affected by the extrapolation method, as shown in Figure 2.

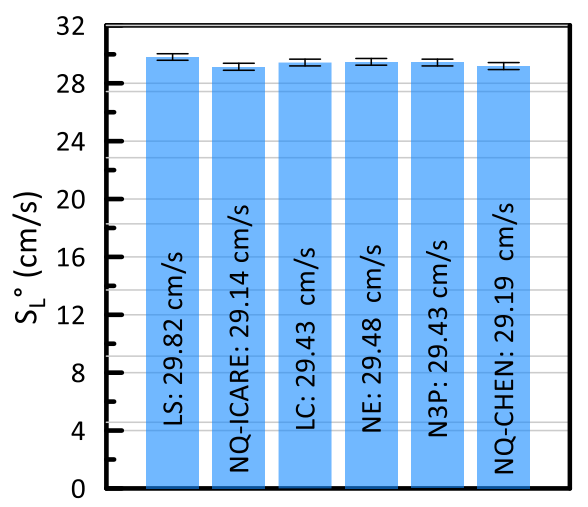

Figure 2. Laminar flame speed at zero-stretch according to the extrapolation method for $\phi=0.93$ at an initial temperature of $318 \mathrm{~K}$. The extrapolation methods are: LS, linear; NQ, quasi-steady non-linear, LC, linear based on curvature; N3P, non-linear model with 3 fitting parameters. For more details, see $[19,20]$.

The lowest radius was chosen to avoid any history from the ignition and the largest to avoid any pressure or wall effects. The observed burned volume is about $0.8 \%$ of the vessel volume, and 
the pressure remains constant during the visualization of the flame. More details can be found in $[19,20]$, schematics of the apparatus and representative flame images are available in Supplemental Material.

3. Experimental results and model comparisons

Note that the effect of impurities was investigated for the shock-tube results, using the work of Urzay et al. [21] to define the level of $\mathrm{H}$ impurities under the temperatures investigated herein. Calculations with and without $\mathrm{H}$ impurities in the mixtures were performed with the model of Alexandrino et al., and no difference in the results between the two mixtures was observed. Provided below are the results of the $\mathrm{CO}$ and $\mathrm{H}_{2} \mathrm{O}$ time history measurements, followed by the results of the characteristic time and laminar flame speed tests.

\subsection{CO measurements}

Figure 3 shows some representative CO time histories at (a) low, (b) mid, and (c) high temperatures, along with model predictions. As can be seen, CO formation starts very rapidly in all cases, and the $\mathrm{CO}$ profiles reach a peak in all but the pyrolysis conditions. It can also be observed in Figure 3 that the low temperature cases for oxidation conditions, mainly $\phi=1.0$ and 2.0, do not reach a peak as it occurs after the shock-tube test-time. The delay for reaching this peak (or the plateau under pyrolysis) decreases as the temperature increases. The model from Hu et al. [8] is over-reactive and significantly over-predicts the amount of $\mathrm{CO}$ in all conditions. The Glaude et al. [7] model is also largely over-predicting $\mathrm{CO}$, but this model is significantly under-reactive. Note that Figure 3 was scaled to the experimental test-time for which the over-predictive behaviour

of Glaude simulations cannot be seen for all cases, due to its under-reactivity; the low and mid 
temperature cases do produce a larger $\mathrm{CO}$ concentration but at a much later time. The two other models, Alexandrino et al. [10] and Sun et al. [9], offer better CO concentration predictions, although compared to the experimental values at the peak or plateau the two models are slightly higher by a factor of about 1.1, where the fuel-lean data was the closest to the model predictions. The Alexandrino model is slightly under-reactive by a factor around 1.3 for all cases but the stoichiometric condition where the model was over-reactive by a factor of 0.8. However, the Sun model tends to be over-reactive by a factor of 0.92 , for all cases except for the stoichiometric case where it is around $56 \%$ faster.

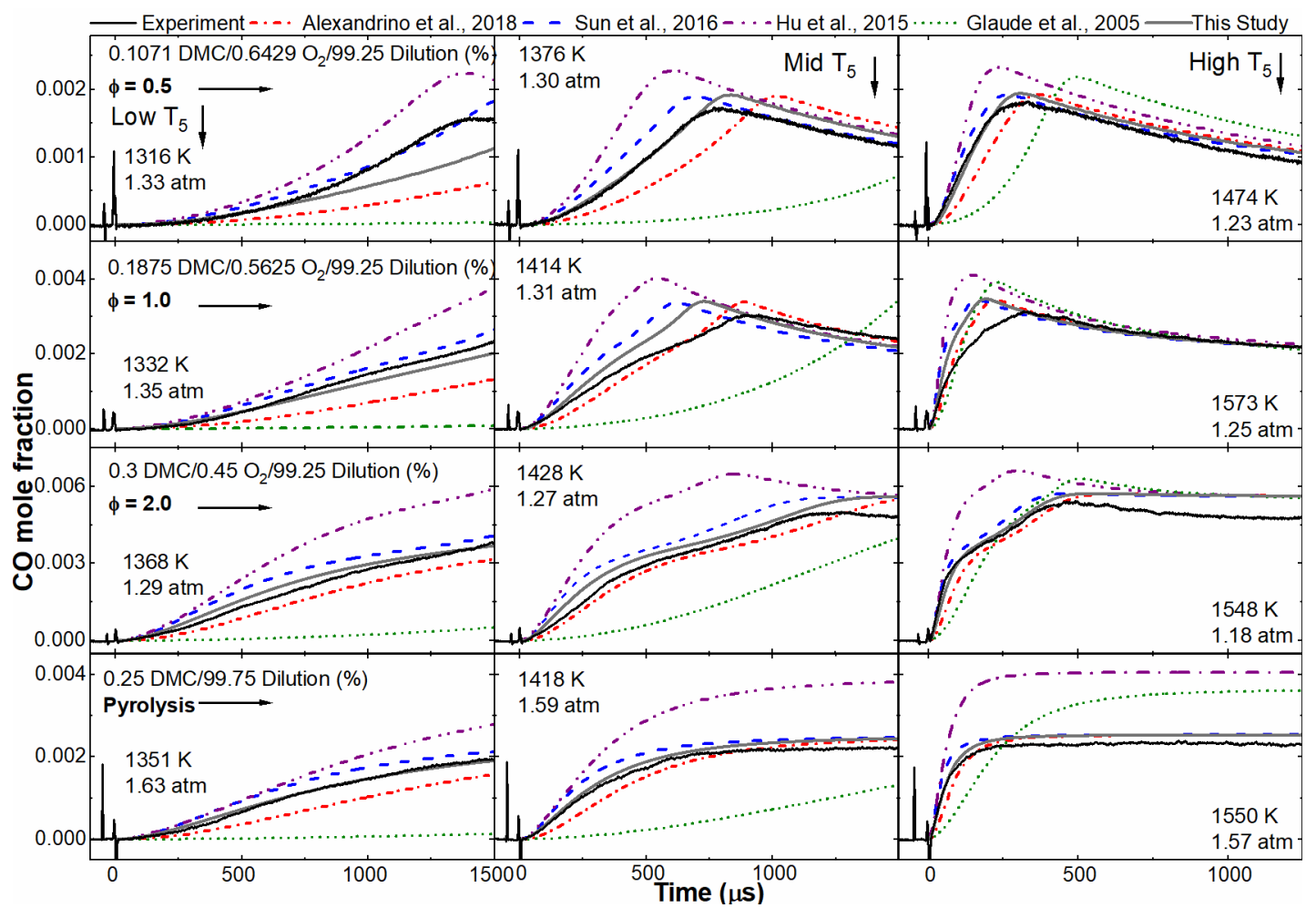

Figure 3. CO time histories and model comparisons for DMC oxidation and pyrolysis.

\section{2. $\mathrm{H}_{2} \mathrm{O}$ measurements}


Experimental and computed $\mathrm{H}_{2} \mathrm{O}$ profiles for the oxidation and pyrolysis cases are presented in Fig. 4. As for CO, representative plots for the low, mid, and high temperatures are provided. For the oxidation cases, all models predict close $\mathrm{H}_{2} \mathrm{O}$ plateau values, in good agreement with the experiments. However, the pyrolysis condition shows that only the Sun and Alexandrino models are able to predict accurately the experimental profiles, the two other models under-predict the amount of water by an estimated factor of 0.7 .

Concerning the reactivity of the models in the presence of oxygen, the Hu and, to a lesser extent, Sun mechanisms are over-reactive except for the fuel-lean, high-temperature condition. The Glaude mechanism is consistently under-reactive, and the model from Alexandrino et al. [10] presents the best predictions overall, despite being under-reactive at low-to-intermediate temperatures.

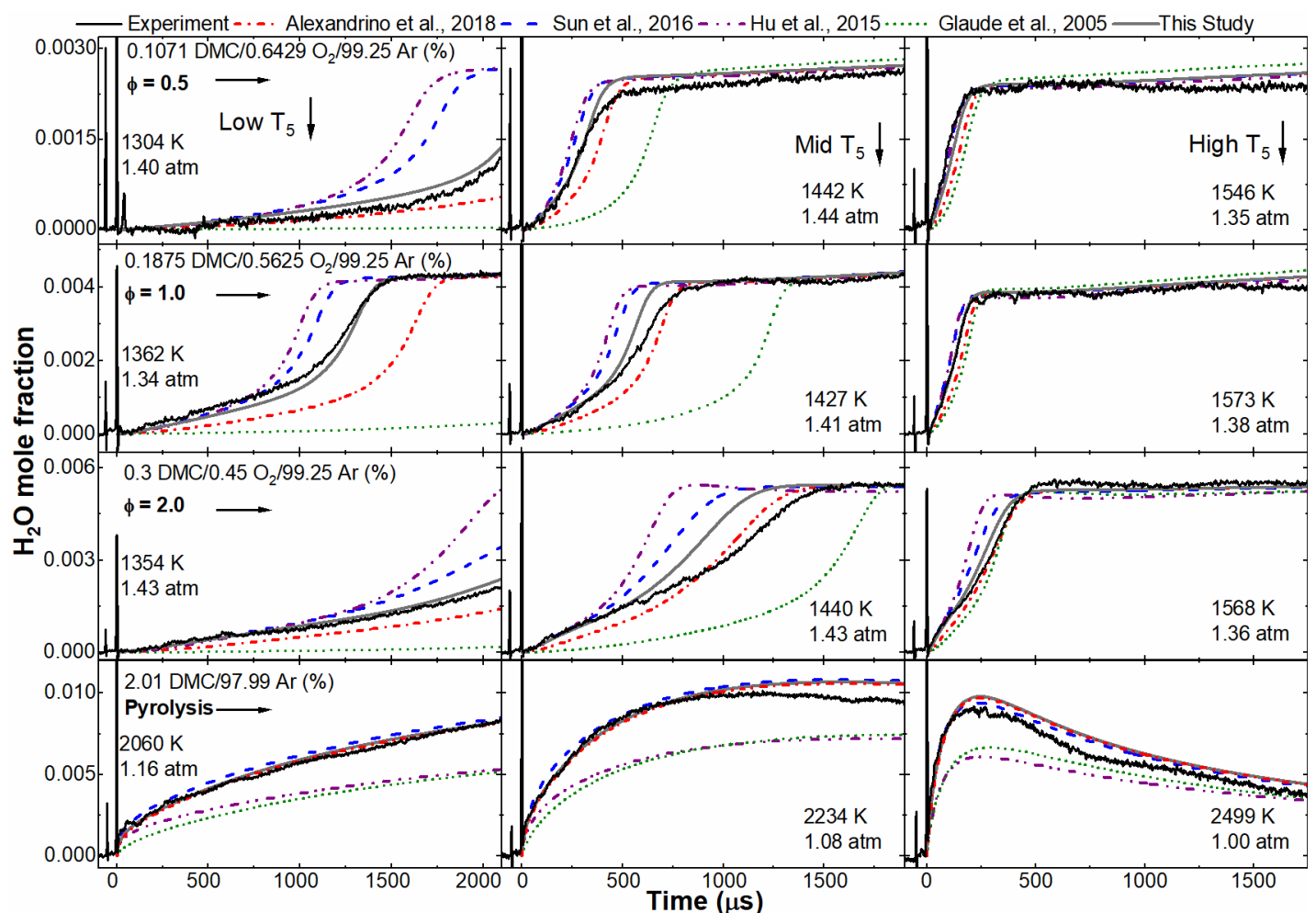

Figure 4. $\mathrm{H}_{2} \mathrm{O}$ time histories and model comparisons for DMC oxidation and pyrolysis. 


\subsection{Characteristic time measurements}

As can be seen in Fig. 5a, $\tau_{\text {peak }}$ increases with $\phi$ and as the temperature decreases. A factor of about 1.5 is found for the time to peak $\mathrm{OH}^{*}$ between the different equivalence ratios on the lowtemperature side, and this factor is increasing with the temperature (a factor up to 2.2 between the fuel-lean and stoichiometric cases at $1475 \mathrm{~K}$ ). The comparison with the models shows that the Glaude model is too slow, notably for the lower-temperature conditions. Predictions from the $\mathrm{Hu}$ and Sun models are close, with the Sun model being closer to the data. These two models predict adequately $\tau_{\text {peak }}$ for the fuel-lean case, but tend to be over-reactive for the other conditions, except for the coldest temperatures investigated. The Alexandrino model is slightly under-reactive at $\phi=$ 0.5 by a factor of 1.5 , but it is relatively accurate most of the time for the other conditions.

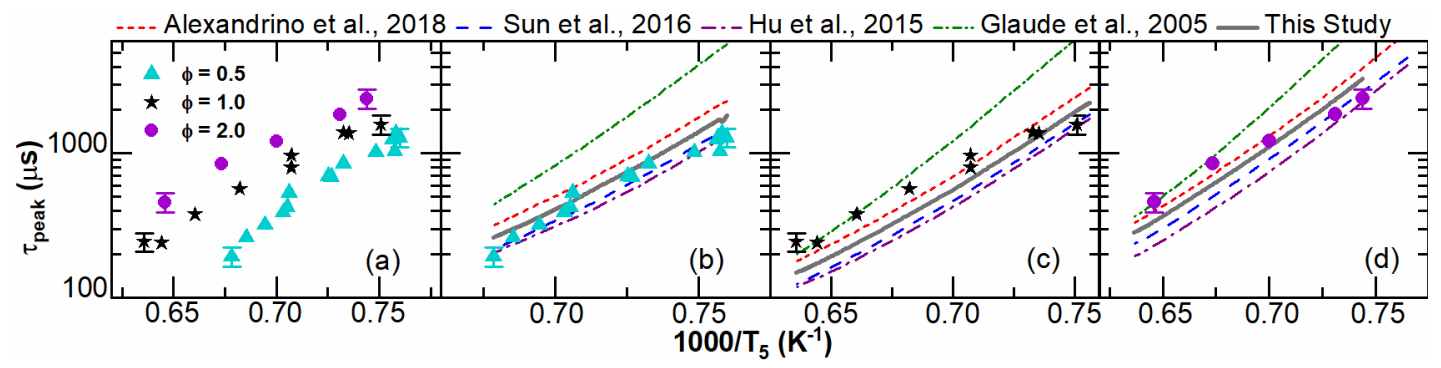

Figure 5. Characteristic reaction times for DMC oxidation (lines: models, symbols: experimental results) (a) experimental results, (b), (c), and (d) model comparison at $\phi=0.5,1.0$, and 2.0, respectively.

\subsection{Flame Speed measurements}

Laminar flame speed experiments were conducted at an initial pressure of 1.013 bar, for equivalence ratios ranging from 0.7 to 1.5 . The limits were chosen to ensure successful ignition 
and to avoid flame wrinkling, respectively. Figure 6a shows the evolution of unstretched laminar flame velocity $\left(S_{L, u}^{0}\right)$ of DMC/air mixtures for several initial temperatures as a function of $\phi$. It is visible that $S_{L, u}^{0}$ notably increases with the initial temperature, with maximum values reached at $\phi=1.1(31.4,38.7$, and $51.4 \mathrm{~cm} / \mathrm{s}$ at 318,363 , and $423 \mathrm{~K}$, respectively).

Results at 318 and $363 \mathrm{~K}$ were compared with data from Bardin et al. [22], measured at 318 and $358 \mathrm{~K}$ on two experimental setups (denoted I and II). The present data are in reasonable agreement with the literature data, with the best agreement obtained with measurements from their first setup. Some discrepancies can be observed with the results from their second setup, with the present $S_{L, u}^{0}$ being consistently slower by about $2 \mathrm{~cm} / \mathrm{s}$. This discrepancy could be explained by various reasons such as the difference in the methods (heat flux burner vs spherical bomb [22]), the initial temperature uncertainty and/or the $\mathrm{O}_{2} / \mathrm{N}_{2}$ ratio between the two types of experiments.

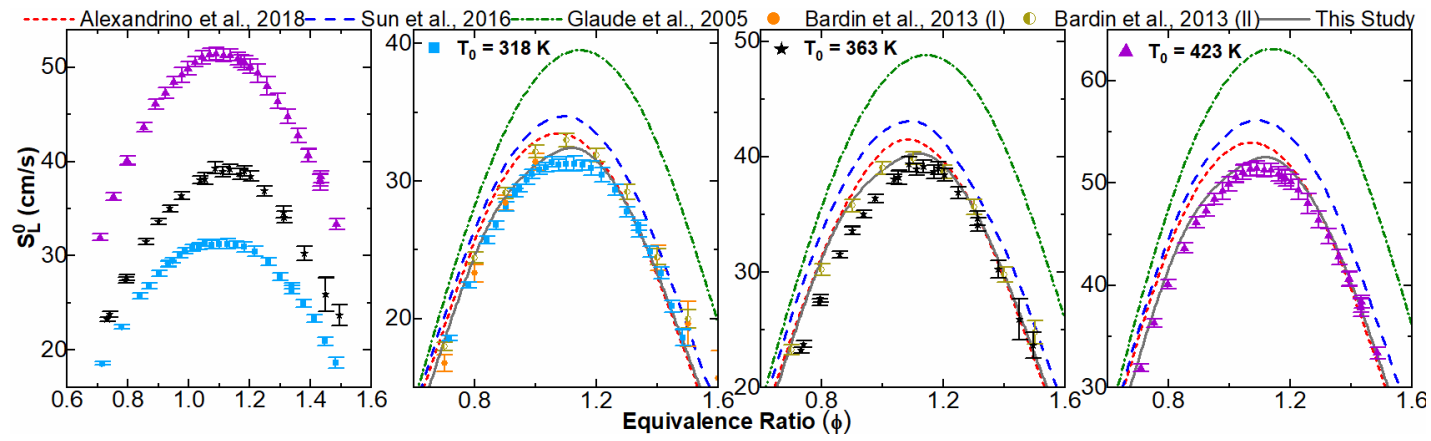

Figure 6. $S_{L, u}^{0}$ for DMC/air mixtures at $\mathrm{P}=1.013$ bar. (Symbols: Experimental data, lines: Models) (a) experimental results, (b), (c), and (d) model comparison at 318, 363, and $423 \mathrm{~K}$, respectively.

Laminar flame speed modelling was performed using the Glaude, Sun, and Alexandrino models only, since no transport data were provided by Hu et al. [8]. As seen in Fig. 6, the models 
are capturing the experimental trends with the change in temperature and equivalence ratio, but they predict flame speeds that are too high in most cases. This observation is indeed the case for the Glaude model, for all conditions. The Sun model also over-predicts the maximum flame speed by a factor of 1.11 , but is only slightly above the experimental error for $318 \mathrm{~K}$ and $423 \mathrm{~K}$ by a factor of 1.15 and 1.09 on the fuel-lean and fuel-rich sides, respectively. For $363 \mathrm{~K}$, Sun model is over-predictive by a factor up to 1.24 on the fuel-lean side, while it shows the least deviation from the experimental data on the fuel-rich side with a factor of 0.87 . Finally, the tentative model shown as "This Study" in the figures (discussed below) is the closest to the data, with the most accurate predictions occurring on the fuel-rich side. As depicted in Figure 6, the tentative model is not only closer to the experimental data than the other models in terms of maximum flame speed, but this maximum is now slightly shifted towards the fuel rich side too, closer to the experiments.

\section{Discussion}

Overall, the results presented herein showed that the model from Alexandrino et al. [10] was

the most accurate. Using this model, a sensitivity analysis was conducted for $S_{L, u}^{0}$, and also for the $\mathrm{CO}$ and $\mathrm{H}_{2} \mathrm{O}$ profiles, for the three studied equivalence ratios and for an intermediate temperature at atmospheric pressure (Fig. 7). A sensitivity analysis was performed for the tentative model, visible in Supplemental Material, showing no significant differences from the overall trends depicted by the Alexandrino et al. mechanism. 

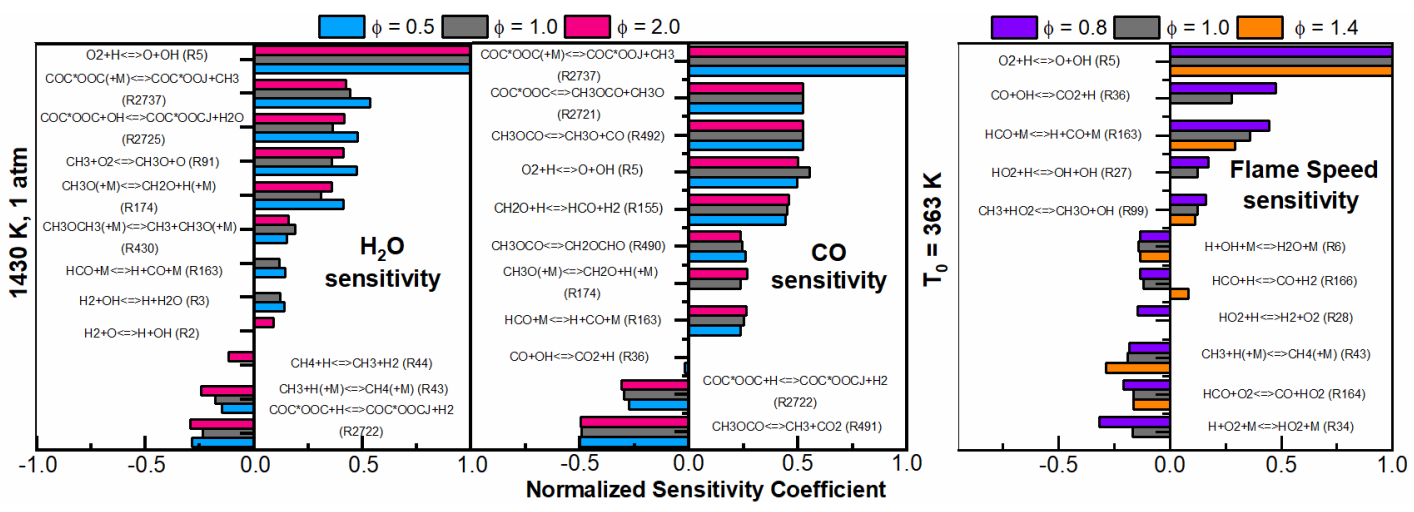

Figure 7. Normalized sensitivity coefficients at intermediate temperature for shock-tube species concentration and laminar flame speed measurements using the model from Alexandrino et al. [10].

For the shock-tube results, besides well-characterized reactions pertaining to the so-called base chemistry (constituting all of the flame speed analysis), this analysis exhibited three important reactions involving DMC $\left(\mathrm{COC}^{*} \mathrm{OOC}\right.$ in the model): $\mathrm{COC} * \mathrm{OOC}(+\mathrm{M}) \leftrightarrows \mathrm{COC}^{*} \mathrm{OO}+\mathrm{CH}_{3}(+\mathrm{M})$ (R2737), $\mathrm{COC} * \mathrm{OOC}+\mathrm{H} \leftrightarrows \mathrm{COC} * \mathrm{OOCj}+\mathrm{H}_{2} \quad(\mathrm{R} 2722)$, and $\mathrm{COC} * \mathrm{OOC}+\mathrm{OH} \leftrightarrows \mathrm{COC} * \mathrm{OOCj}+\mathrm{H}_{2} \mathrm{O}$ (R2725), referring to the reaction numbers corresponding to the Alexandrino et al. model, which uses AramcoMech 2.0 as its base mechanism. To demonstrate how modifications to these three reactions could improve the predictions, a tentative model was assembled by starting with the updated AramcoMech 3.0 [23] and Alexandrino et al. mechanisms. The reactions R2722, R2725, and R2737, were then modified by adjusting their pre-exponential factor, with the present data as targets. To summarize, the optimum solution was found with the following adjustments of the preexponential factors: $\mathrm{k}_{\mathrm{R} 2737} \times 1.54, \mathrm{k}_{\mathrm{R} 2722} / 2$, and $\mathrm{k}_{\mathrm{R} 2725} \times 3$. Results from this tentative model are presented in Figs. 3-7. The resulting predictions are notably improved for both the shock-tube and $S_{L, u}^{0}$ results. These adjustments can be justified by the fact that the reaction rate of specific reactions in the DMC submechanism are not very well characterized, as detailed below. 
$\mathrm{R} 2737\left(\mathrm{COC}^{*} \mathrm{OOC}(+\mathrm{M}) \leftrightarrows \mathrm{COC} * \mathrm{OOj}+\mathrm{CH}_{3}(+\mathrm{M})\right)$ is of primary importance for shock-tube results, and Alexandrino et al. utilized the reaction rate proposed by Dooley et al. [27], estimated for a similar reaction with methyl-butanoate [27] (based on $\tau_{i g n}$ measurements), on the account that the reaction rate is in "reasonable agreement" with the one determined by Peukert et al. [25] for DMC and that methyl-butanoate presents a similar chemical structure. For reaction R2722 $\left(\mathrm{COC} * \mathrm{OOC}+\mathrm{H} \leftrightarrows \mathrm{COC} * \mathrm{OOCj}+\mathrm{H}_{2}\right)$, Alexandrino et al. used the rate employed in Sun et al. [9], which is the reaction rate proposed by Peukert and coworkers [25]. Finally, for R2725 $\left(\mathrm{COC} * \mathrm{OOC}+\mathrm{OH} \leftrightarrows \mathrm{COC} * \mathrm{OOCj}+\mathrm{H}_{2} \mathrm{O}\right)$, Alexandrino et al. used the rate proposed by Sun et al., which has been assessed by analogy with a similar reaction with methyl-formate [24]. Note that R2725 has been recently studied by Khaled et al. [26], both experimentally and by high-level calculation. Two corresponding reaction rates were determined near $1.5 \mathrm{~atm}$. The reaction rate deriving from the experimental data was determined between 872 and $1295 \mathrm{~K}$ whereas the calculated reaction rate was determined in the $300-1500 \mathrm{~K}$ range. A graph of the evolution of the various reaction rates for R2725 versus the temperature is visible in Sup. Mat.

Note that for the highest temperature investigated herein, $2500 \mathrm{~K}$, the reaction rate proposed in this study is close to the calculated rate from Khaled et al., only $14 \%$ higher, and it is worth mentioning that the experimental rate is also slightly higher than the value used in the Alexandrino model. On the low temperature side of our conditions, the experimental rate of Khaled et al. and the one from the Alexandrino model are close, within 13\%, making the rate for the tentative model 3 times higher overall. Note also that the calculated rate significantly diverges at this low temperature. It is worth mentioning that the rates from Khaled et al. were both alternatively used 
in the tentative model and the Alexandrino model, and none were improving the predictions (Fig. SM 14-15).

In the authors' opinion, this discussion is a good indicator of the future work that is necessary to better determine directly, either experimentally and/or by high-level calculations, the rate of the few reactions that involve DMC, and, as such, the tentative model cannot be used to confidently model DMC chemistry.

\section{Conclusions}

New global $\left(\tau_{\text {peak }}, S_{L, u}^{0}\right)$ and fundamental $\left(\mathrm{H}_{2} \mathrm{O}\right.$ and $\mathrm{CO}$ time histories $)$ experiments were performed to investigate DMC combustion chemistry near atmospheric pressure. Few kinetics data are available in the literature for DMC, and the species time histories and laminar flame speed data obtained in the present study are the first of their kind for DMC, to the authors' knowledge. These data were compared to several detailed kinetics models from the literature. None of the models however were able to adequately predict the data over the entire range of conditions investigated, and the new data obtained herein can be used to improve the chemistry of DMC combustion.

Sensitivity and reaction pathway analyses were performed with the most-accurate model, allowing the identification of key reactions involving DMC. The rate of these reactions was reasonably modified, and a tentative model was proposed. Despite the fact the flame speed results were in general sensitive to well-characterized reactions from the base chemistry, the changes in the DMC reactions did have an impact on the flame speed results but to a lesser extent than the shock-tube data. It was found possible to greatly improve the predictions by using this tentative model, indicating a great need to further study the rate of the three reactions modified. 


\section{Acknowledgments}

The shock-tube portion of this work was funded by J. Mike '66 Department of Mechanical Engineering and the TEES Turbomachinery Laboratory. Funding for the flame speed experiments came from the "Conseil Régional du Centre-Val de Loire", France. 


\section{References}

[1] A.H. Tamboli, A.A. Chaugule, H. Kim, Catalytic developments in the direct dimethyl carbonate synthesis from carbon dioxide and methanol, Chem. Eng. J., 323 (2017) 530544.

[2] M.U. Alzueta, P. Salinas, Á. Millera, R. Bilbao, M. Abián, A study of dimethyl carbonate conversion and its impact to minimize soot and NO emissions, Proc. Combust. Inst., 36 (2017) 3985-3993.

[3] A.O.G. Abdalla, D. Liu, Dimethyl carbonate as a promising oxygenated fuel for combustion: A review, Energies, 11 (2018) 1552.

[4] Q. Wang, B. Mao, S.I. Stoliarov, J. Sun, A review of lithium ion battery failure mechanisms and fire prevention strategies, Prog. Energy Combust. Sci., 73 (2019) 95-131.

[5] S.J. Harris, A. Timmons, W.J. Pitz, A combustion chemistry analysis of carbonate solvents used in Li-ion batteries, J. Pow. Sources, 193 (2009) 855-858.

[6] Y. Fernandes, A. Bry, S. de Persis, Identification and quantification of gases emitted during abuse tests by overcharge of a commercial Li-ion battery, J. Pow. Sources, 389 (2018) 106119.

[7] P.A. Glaude, W.J. Pitz, M.J. Thomson, Chemical kinetic modeling of dimethyl carbonate in an opposed-flow diffusion flame, Proc. Combust. Inst., 30 (2005) 1111-1118.

[8] E. Hu, Y. Chen, Z. Zhang, L. Pan, Q. Li, Y. Cheng, Z. Huang, Experimental and kinetic study on ignition delay times of dimethyl carbonate at high temperature, Fuel, 140 (2015) 626-632.

[9] W. Sun, B. Yang, N. Hansen, C.K. Westbrook, F. Zhang, G. Wang, K. Moshammer, C.K. Law, An experimental and kinetic modeling study on dimethyl carbonate (DMC) pyrolysis and combustion, Combust. Flame, 164 (2016) 224-238.

[10] K. Alexandrino, M.U. Alzueta, H.J. Curran, An experimental and modeling study of the ignition of dimethyl carbonate in shock tubes and rapid compression machine, Combust. Flame, 188 (2018) 212-226.

[11] E.L. Petersen, M.J.A. Rickard, M.W. Crofton, E.D. Abbey, M.J. Traum, D.M. Kalitan, A facility for gas- and condensed-phase measurements behind shock waves, Meas. Sci. and Tech., 16 (2005) 1716-1729.

[12] O. Mathieu, C.R. Mulvihill, E.L. Petersen, Assessment of modern detailed kinetics mechanisms to predict $\mathrm{CO}$ formation from methane combustion using shock-tube laserabsorption measurements, Fuel, 236 (2019) 1164-1180. 
[13] L.S. Rothman, D. Jacquemart, A. Barbe, D. Chris Benner, M. Birk, L.R. Brown, M.R. Carleer, C. Chackerian, K. Chance, L.H. Coudert, V. Dana, V.M. Devi, J.M. Flaud, R.R. Gamache, A. Goldman, J.M. Hartmann, K.W. Jucks, A.G. Maki, J.Y. Mandin, S.T. Massie, J. Orphal, A. Perrin, C.P. Rinsland, M.A.H. Smith, J. Tennyson, R.N. Tolchenov, R.A. Toth, J. Vander Auwera, P. Varanasi, G. Wagner, The HITRAN 2004 molecular spectroscopic database, J. Quant. Spectros. and Radiative Transfer, 96 (2005) 139-204.

[14] W. Ren, A. Farooq, D.F. Davidson, R.K. Hanson, CO concentration and temperature sensor for combustion gases using quantum-cascade laser absorption near $4.7 \mu \mathrm{m}$, App. Phys. B, 107 (2012) 849-860.

[15] V. Nagali, D.F. Davidson, R.K. Hanson, Measurements of temperature-dependent argonbroadened half-widths of $\mathrm{H}_{2} \mathrm{O}$ transitions in the $7117 \mathrm{~cm}^{-1}$ region, J. Quant. Spectros. and Radiative Transfer, 64 (2000) 651-655.

[16] C.R. Mulvihill, C.L. Keesee, T. Sikes, R.S. Teixeira, O. Mathieu, E.L. Petersen, Ignition delay times, laminar flame speeds, and species time-histories in the $\mathrm{H}_{2} \mathrm{~S} / \mathrm{CH}_{4}$ system at atmospheric pressure, Proc. Combust. Inst., 37 (2019) 735-742.

[17] O. Mathieu, C. Mulvihill, E.L. Petersen, Shock-tube water time-histories and ignition delay time measurements for H2S near atmospheric pressure, Proc. Combust. Inst., 36 (2017) 4019-4027.

[18] P.D. Ronney, On the mechanisms of flame propagation limits and extinguishmentprocesses at microgravity, Proc. Combust. Inst., 22 (1989) 1615-1623.

[19] R. Grosseuvres, A. Comandini, A. Bentaib, N. Chaumeix, Combustion properties of $\mathrm{H}_{2} / \mathrm{N}_{2} / \mathrm{O}_{2} /$ steam mixtures, Proc. Combust. Inst., 37 (2019) 1537-1546.

[20] D. Nativel, M. Pelucchi, A. Frassoldati, A. Comandini, A. Cuoci, E. Ranzi, N. Chaumeix, T. Faravelli, Laminar flame speeds of pentanol isomers: An experimental and modeling study, Combust. Flame, 166 (2016) 1-18.

[21] J. Urzay, N. Kseib, D.F. Davidson, G. Iaccarino, R.K. Hanson, Uncertainty-quantification analysis of the effects of residual impurities on hydrogen-oxygen ignition in shock tubes, Combust. Flame, 161 (2014) 1-15.

[22] M.E. Bardin, E.V. Ivanov, E.J.K. Nilsson, V.A. Vinokurov, A.A. Konnov, Laminar burning velocities of dimethyl carbonate with air, Energy \& Fuels, 27 (2013) 5513-5517.

[23] C.-W. Zhou, Y. Li, U. Burke, C. Banyon, K.P. Somers, S. Ding, S. Khan, J.W. Hargis, T. Sikes, O. Mathieu, E.L. Petersen, M. AlAbbad, A. Farooq, Y. Pan, Y. Zhang, Z. Huang, J. Lopez, Z. Loparo, S.S. Vasu, H.J. Curran, An experimental and chemical kinetic modeling study of 1,3-butadiene combustion: Ignition delay time and laminar flame speed measurements, Combust. Flame, 197 (2018) 423-438.

[24] S. Dooley, M.P. Burke, M. Chaos, Y. Stein, F.L. Dryer, V.P. Zhukov, O. Finch, J.M. Simmie, H.J. Curran, Methyl formate oxidation: Speciation data, laminar burning 
velocities, ignition delay times, and a validated chemical kinetic model, International Journal of Chemical Kinetics, 42 (2010) 527-549.

[25] S.L. Peukert, R. Sivaramakrishnan, J.V. Michael, High Temperature Shock Tube and Theoretical Studies on the Thermal Decomposition of Dimethyl Carbonate and Its Bimolecular Reactions with H and D-Atoms, J. Phys. Chem. A, 117 (2013) 3718-3728.

[26] F. Khaled, B.R. Giri, M. Szőri, T.V.T. Mai, L.K. Huynh, A. Farooq, A combined hightemperature experimental and theoretical kinetic study of the reaction of dimethyl carbonate with OH radicals, Physical Chemistry Chemical Physics, 19 (2017) 7147-7157.

[27] S. Dooley, H.J. Curran, J.M. Simmie, Autoignition measurements and a validated kinetic model for the biodiesel surrogate, methyl butanoate, Combust. Flame, 153 (2008) 2-32. 